\title{
EVIDENCE OF MULTIEXPONENTIAL $T_{2}$ IN RAT GLIOBLASTOMA
}

\author{
Richard D. Dortch ${ }^{1,2}$, Thomas E. Yankeelov ${ }^{1,2,3,4,5}$, Zoe Yue ${ }^{2}$, Christopher C. Quarles ${ }^{1,2,3}$, \\ John C. Gore ${ }^{1,2,3,4,6}$, and Mark D. Does ${ }^{1,2,3}$ \\ ${ }^{1}$ Biomedical Engineering, Vanderbilt University, Nashville, TN, United States \\ ${ }^{2}$ Institute of Imaging Science, Vanderbilt University, Nashville, TN, United States \\ ${ }^{3}$ Radiology and Radiological Sciences, Vanderbilt University, Nashville, TN, United States \\ ${ }^{4}$ Physics and Astronomy, Vanderbilt University, Nashville, TN, United States \\ ${ }^{5}$ Cancer Biology, Vanderbilt University, Nashville, TN, United States \\ ${ }^{6}$ Molecular Physiology and Biophysics, Vanderbilt University, Nashville, TN, United States
}

\begin{abstract}
The aim of this study was to characterize multiexponential $T_{2}\left(\mathrm{MET}_{2}\right)$ relaxation in a rat $\mathrm{C} 6$ glioblastoma tumor model. To do this, rats $(\mathrm{n}=11)$ were inoculated with the C6 cells via stereotaxic injection into the brain. Ten days later, $\mathrm{MET}_{2}$ measurements were performed in vivo using a single-slice, multi-echo spin-echo sequence at 7.0 T. Tumor signal was biexponential in eight animals with a short-lived $T_{2}$ component $\left(T_{2}=20.7 \pm 5.4 \mathrm{~ms}\right.$ across samples) representing $6.8 \pm 6.2 \%$ of the total signal and a long-lived $T_{2}$ component $\left(T_{2}=76.4 \pm 9.3 \mathrm{~ms}\right)$ representing the remaining signal fraction. In contrast, signal from contralateral grey matter was consistently monoexponential $\left(T_{2}=48.8 \pm 2.3 \mathrm{~ms}\right)$. Additional ex vivo studies $(\mathrm{n}=3)$ and Monte Carlo simulations showed that the in vivo results were not significantly corrupted by partial volume averaging or noise. The underlying physiological origin of the observed $\mathrm{MET}_{2}$ components is unknown; however, $\mathrm{MET}_{2}$ analysis may hold promise as a non-invasive tool for characterizing tumor microenvironment in vivo on a sub-voxel scale.
\end{abstract}

\section{Keywords}

MRI; multiexponential $T_{2}$; relaxometry; brain; tumor

\section{INTRODUCTION}

The nuclear magnetic resonance (NMR) signal has been shown to exhibit multiexponential $T_{2}\left(\mathrm{MET}_{2}\right)$ decay in a number of tissues (e.g., white matter $(1,2)$, peripheral nerve $(3,4)$, skeletal muscle (5)), arising from a combination of microanatomical water compartition (e.g., intracellular and extracellular water) and slow intercompartmental exchange. The goal

Please address correspondence to: Mark D. Does, Ph.D., Vanderbilt University Institute of Imaging Science, $116121^{\text {st }}$ Avenue South, AA 1105 MCN, Nashville, Tennessee 37232-2310, mark.does@ vanderbilt.edu, Phone: 615-322-8352, Fax: 615-322-0734.

This work was presented, in part, at the International Society for Magnetic Resonance in Medicine $16^{\text {th }}$ Scientific Meeting and Exhibition, Toronto, 2008. 
of $\mathrm{MET}_{2}$ analysis is to decompose bulk NMR signal into components that represent underlying tissue compartments. In doing so, one can probe individual tissue compartments that exist on a sub-voxel scale.

Given the heterogeneous nature of tumors, one might expect to observe $\mathrm{MET}_{2}$ relaxation in tumors. In fact, $\mathrm{MET}_{2}$ relaxation has been observed in animal models (both in vivo (6) and ex vivo (7)), surgical samples ex vivo $(8,9)$, and in humans in vivo (10), though results have been inconsistent. This inconsistency is presumably a product of differences in tumor types, models, and staging as well as differences in the data acquisition and analysis techniques used. Of these previous studies, only one (10) attempted to characterize $\mathrm{MET}_{2}$ relaxation in tumors using an imaging approach (others used non-imaging CPMG $(11,12)$ approaches). In that particular study, $T_{2}$ measurements were made in human gliomas and the resultant signal was found to decay biexponentially; however, as the authors note, these results should be interpreted carefully due to the low signal-to-noise ratio (SNR) of the data.

The goal of this study was to characterize $\mathrm{MET}_{2}$ relaxation in a rat glioblastoma tumor model. Towards this end, we performed Monte Carlo simulations as well as in vivo and ex vivo imaging experiments. The simulations were designed to see if the experimental imaging data achieved the appropriate SNR to perform region of interest (ROI) and/or voxel-based analyses. The in vivo data were acquired to characterize $\mathrm{MET}_{2}$ relaxation in a commonly used tumor model, while the ex vivo measurements were acquired to ensure that the in vivo results were not significantly corrupted due to either partial-voluming or low SNR.

\section{EXPERIMENTAL}

\section{Simulations}

Monte Carlo simulations were performed to determine the SNR requirements for $\mathrm{MET}_{2}$ analysis of tumor signal. $\mathrm{MET}_{2}$ decay curves, $y_{i}$ were generated according to

$$
y_{i}=\sum_{j=1}^{3}\left|s_{j} \exp \left(-t_{i} / T_{2, j}\right)+\varepsilon_{i}\right|, \quad i=1,2, \ldots, \mathrm{NE}
$$

where $s_{j}$ is the weight of the exponential component associated with $T_{2, j}, \mathrm{NE}$ is the total number of echoes, $t_{i}$ is the echo time (TE) of the $i^{\text {th }}$ echo, and $\varepsilon_{i}$ is Gaussian noise added to the $i^{\text {th }}$ echo.

Two sets of simulations were generated using Eq. [1] and the mean experimental values listed in Table 1. The first set was designed to simulate the biexponential decay observed in vivo (see Table 2), while the second set was designed to simulate the triexponential decay observed ex vivo (see Table 3). The decay curve sampling for each set was designed to match its corresponding decay curve sampling during experimental studies. Within each set, decay curves were generated with SNR values logarithmically spaced between 100 and 3200, where SNR is defined as the magnitude of the first echo divided by the standard deviation of the Gaussian noise. For a given SNR, 2000 decay curves (each with a unique noise realization) were generated and the bias introduced by the magnitude operation, which 
converts the underlying Gaussian noise to Rician, was removed (see Data Analysis for more details) (13). $\mathrm{MET}_{2}$ analysis was then performed to determine the distribution of $T_{2} \mathrm{~s}$ (i.e., the $T_{2}$ spectrum) in the simulated data. This was done by minimizing

$$
\sum_{i=1}^{\mathrm{NE}}\left\{\sum_{j=1}^{J}\left(s_{j} \exp \left(-t_{i} / T_{2, j}\right)-y_{i}\right)^{2}\right\}+\mu \sum_{j=2}^{J-1}\left(s_{j-1}-2 s_{j}+s_{j+1}\right)^{2}
$$

in a non-negative least-squares (NNLS) (14) sense using $J=100$ logarithmically spaced relaxation times over the interval $t_{1} / 2<T_{2, j}<2 t_{N E}$. The first term in Eq. [2] minimizes data misfit, while the second term regularizes the solution by imposing a minimum curvature constraint (15), which smoothes the resultant spectrum according to the magnitude of the regularizer term, $\mu$. For this study, the value of $\mu$ was determined in a two-step process. First, $\mu$ was iteratively updated to determine the value that increased the $\chi^{2}$ misfit by $2 \%$ relative to the unregularized fit $(\mu=0)$ for each unique decay curve (16). Second, the median $\mu$ across data with the same SNR was determined, and was subsequently used in a final regularized fit so that all $T_{2}$ spectra from data with the same SNR were smoothed to the same degree.

Each spectral peak, $n$, and its corresponding range of relaxation times $T_{2, n \text {, min }}<T_{2, j}$ $<T_{2, n \text {,max }}$, was identified using an automated peak- and valley-finding algorithm (based upon locating zero-crossings of the first-derivative of the spectrum). For all non-spurious components (defined here as any peak that was consistently observed and represented more than $1 \%$ the total signal), relaxation times and signal fractions were then calculated from

$$
\bar{T}_{2, n}=\frac{\sum_{j=n, \min }^{n, \max } T_{2, j} s_{j}}{\sum_{j=n, \min }^{n, \max } s_{j}}
$$

and

$$
p_{n}=\frac{\sum_{j=n, \min }^{n, \max } s_{j}}{\sum_{j=1}^{J} s_{j}},
$$

respectively. Spectra were deemed admissible if the expected components (two for in vivo and three for ex vivo simulations, respectively) were resolved. The percentage of admissible spectra, or admissibility, was then calculated for each SNR value to determine the SNR requirements for $\mathrm{MET}_{2}$ analysis. 


\section{Animal Preparation}

All procedures were approved by our Institution's Animal Care and Usage Committee. Male Wistar rats $(\mathrm{n}=11)$ were immobilized and anesthesia was induced and maintained with a $2 \% / 98 \%$ isofluorane/oxygen mixture (Minrad Inc., Bethlehem, PA). Rats were then inoculated with $1 \times 10^{5} \mathrm{C} 6$ gliosarcoma cells using a $10-\mu \mathrm{L}$ gastight syringe approximately one $\mathrm{mm}$ anterior and two $\mathrm{mm}$ lateral to bregma on the right side of the head, at a depth of three $\mathrm{mm}$ relative to the dural surface. C6 is a common glioblastoma model that is widely used in experimental neuro-oncology to evaluate tumor growth, invasion, migration, and blood-brain-barrier disruptions, and has been used extensively to investigate the efficacy of various therapies including chemotherapy and radiation therapy (17). In vivo MRI was performed 14 to 21 days (see Table 2 for details) after this procedure.

After in vivo imaging, a transcardial perfusion was performed in three of the eleven animals described above. During this procedure, animals were immobilized and anesthesia was induced and maintained with a $2 \% / 98 \%$ isofluorane/oxygen mixture. A 23-g needle was inserted into the left ventricle of the heart and phosphate-buffered saline (PBS; Mediatech Inc., Herdon, VA) was perfused to flush the blood out of the vascular system, at which point the perfusate was changed to $10 \%$ neutral buffered formalin (VWR, West Chester, PA). Following perfusion, brains were extracted and stored in 10\% neutral buffered formalin overnight. Brains were then stored in PBS (to remove excess fixative) until ex vivo MRI was performed.

\section{In vivo Magnetic Resonance}

Rats $(\mathrm{n}=11)$ were imaged using a 7.0-T, 16-cm bore Varian INOVA spectrometer (Varian Inc., Palo Alto, CA). A 63-mm inner diameter quadrature volume coil was used for RF transmission and reception. During imaging, animals were anesthetized with a 2\%/98\% isofluorane/oxygen mixture and temperature was maintained at $37^{\circ} \mathrm{C}$ with a rectal thermometer and warm-air feedback system. Respiratory signals were also monitored using a small pneumatic pillow placed near the rat's abdomen (SA Instruments, Stony Brook, NY).

For each rat, a single 1.5-mm axial slice through the center of the tumor volume was first selected from $T_{2}$-weighted scout images. $T_{2}$ measurements were then made using a singleslice, multi-echo spin-echo sequence (18) with an echo spacing $(\Delta \mathrm{TE})=8 \mathrm{~ms}, \mathrm{TR}=4 \mathrm{~s}$, number of echoes $(\mathrm{NE})=32$, field of view $=35 \times 35 \mathrm{~mm}^{2}$, acquisition matrix $=64 \times 64$, inplane resolution $=547 \times 547 \mu \mathrm{m}^{2}$, acquisition bandwidth $=50 \mathrm{kHz}$, number of averages $(\mathrm{NEX})=8$, and total imaging time $\approx 34$ minutes. Spoiler gradients were placed about each $90_{\mathrm{x}} 180_{\mathrm{y}} 90_{\mathrm{x}}$ broadband composite refocusing pulse (19) in an alternating and descending fashion (18) in order to remove signal from unwanted coherence pathways. The spoiler gradients were calculated so as to cause a minimum phase dispersion of $\pi / 2$ across one slice thickness, which was found to be sufficiently large to remove these unwanted signal components. 


\section{Ex vivo Magnetic Resonance}

Excised brains $(\mathrm{n}=3)$ were imaged at bore temperature $\left(\approx 20{ }^{\circ} \mathrm{C}\right)$ using a 7.0-T, 16-cm bore Varian INOVA spectrometer and 38-mm diameter Litz RF coil (Doty Scientific, Columbia, SC). For imaging, brains were placed in a custom-built holder and bathed in fresh PBS. To minimize air bubbles, both the brain and holder were fully immersed in PBS while loading the brain into the holder.

A single 1-mm axial slice through the center of the tumor volume (representing approximately the same slice acquired in vivo) was first selected from $T_{2}$-weighted scout images (Fig. 1b). Imaging was then performed using the same multiple-spin echo sequence as described for in vivo imaging, but at higher resolution and SNR. Relevant parameters for ex vivo $T_{2}$ measurements included $\Delta \mathrm{TE}=15 \mathrm{~ms}, \mathrm{TR}=4 \mathrm{~s}, \mathrm{NE}=40$, field of view $=19.2 \times$ $19.2 \mathrm{~mm}^{2}$, acquisition matrix $=64 \times 64$, in-plane resolution $=300 \times 300 \mu \mathrm{m}^{2}$, acquisition bandwidth $=40 \mathrm{kHz}, \mathrm{NEX}=64$, and total imaging time $\approx 4 \frac{1}{1} 2$ hours. Note the echo spacing used for ex vivo acquisitions (15 ms) was longer than for in vivo acquisitions (8 ms). This was to ensure that the decay curve was adequately sampled ex vivo because: 1) the observed relaxation times ex vivo were generally longer than corresponding relaxation times in vivo and 2) an additional long-lived component was observed ex vivo (see Results).

\section{Data Analysis}

All data analysis was performed using routines written in-house in MATLAB (Mathworks, Natick, MA). For ROI-based analysis, tumor and contralateral subcortical grey matter ROIs were defined from $T_{2}$-weighted scout images (as shown in Fig. 1). Tumor ROIs were defined to encompass as much of the tumor as possible, while avoiding the tumor margin where significant partial-voluming of tumor and normal tissue may occur. Once defined, the maximum-likelihood estimator of each Rician-distributed ROI value was determined (20). This approach has been shown to minimize bias introduced by the Rician noise present in magnitude MR images, especially for those images acquired with low SNR (i.e., at longer echo times). Data were then fitted to Eq. [2] in the same manner described for simulated data. For these fits, the regularizer parameter, $\mu$, was iteratively updated to determine the value that increased the $\chi^{2}$ misfit by $2 \%$ relative to the unregularized fit for each individual ROI. The median $\mu$ across samples -0.15 and 0.01 for in vivo and ex vivo data, respectively - was then determined and used in a final regularized fit. Component relaxation times and signal fractions were calculated for each non-spurious component in the resultant $T_{2}$ spectra from Eqs. [3] and [4], respectively.

Simulations showed (see Results for details) that the SNR per voxel was insufficient to perform voxel-based $\mathrm{MET}_{2}$ analysis. Therefore, to increase $\mathrm{SNR}$, in vivo and ex vivo images were spatially filtered by applying a multichannel (with each echo representing a different channel) anisotropic diffusion filter (21) prior to voxel-based $\mathrm{MET}_{2}$ analysis. This approach has been previously applied to $\mathrm{MET}_{2}$ analysis (22) and has been shown to reduce variability with minimal introduction of bias due to partial-voluming. Filtered data were then fitted to Eq. [2] with $\mu=0.07$ and 0.01 for in vivo and ex vivo data, respectively (determined using an analogous approach to that used for ROI-based analyses) in order to determine the $T_{2}$ 
spectrum of each individual voxel and component relaxation times (Eq. [3]) and signal fractions (Eq. [4]) were calculated.

All values are given as mean $( \pm \mathrm{SD})$ across samples unless otherwise reported.

\section{RESULTS}

\section{Simulations}

Fig. 2a displays the admissibility of $\mathrm{MET}_{2}$ analysis for in vivo and ex vivo simulated data as a function of SNR. For ex vivo data, the admissibility for resolving all three observed components as well as resolving the short-lived component from the two longer-lived components was determined. Once again, simulated data were generated using Eq. [1] along with the parameters listed in Table 1.

Assuming a minimum admissibility threshold of $90 \%$, an SNR of approximately 500 is required for in vivo acquisition with two components. The SNR for tumor ROIs was $839 \pm$ 381 (and $649 \pm 211$ for grey matter ROIs), while the SNR per voxel was $193 \pm 61$. Based upon the simulations shown in Fig. 2a, this SNR is sufficient to perform ROI-based MET 2 analysis in vivo; however, SNR is insufficient to perform voxel-based $\mathrm{MET}_{2}$ analysis (with an admissibility of $\approx 50 \%$ ). As a result, spatial smoothing with an anisotropic diffusion filter (as described in Methods) was used to increase SNR for voxel-based $\mathrm{MET}_{2}$ analysis in vivo.

Again assuming a minimum admissibility threshold of $90 \%$, an SNR of approximately 3000 is required to resolve all three components from the ex vivo data. The SNR for tumor ROIs was $3989 \pm 2265$ (and $2335 \pm 344$ for grey matter ROIs), while the SNR per voxel was 557 \pm 28 . Therefore, from Fig. 2 it can be seen that the SNR is sufficient to perform ROI-based $\mathrm{MET}_{2}$ analysis and insufficient to perform voxel-based $\mathrm{MET}_{2}$ analysis ex vivo. As a result, spatial smoothing with an anisotropic filter was also used to increase SNR for voxel-based $\mathrm{MET}_{2}$ analysis ex vivo. Additional simulations showed that an SNR of approximately 1000 is required to resolve the short-lived component (component I) from the two longer-lived components (components II and III) in the ex vivo data. Thus, for comparison to the twocomponent in vivo spectra, ex vivo spectra that exhibited either two or three components were deemed admissible for voxel-based analysis.

An additional set of simulations was performed to assess the effect of the size of the shortlived component $\left(p_{I}\right)$ on admissibility. Sets of two component data were generated over a range of $p_{I}$ values using the same relaxation times and data sampling parameters as the in vivo simulations and an SNR of 800 (similar to ROI-based analysis in vivo). As seen in Fig, $2 \mathrm{~b}$, admissibility was found to increase with increasing size of the short-lived component. Using the same minimum admissibility threshold of $90 \%$ as above, these simulations suggest that we should consistently observe $\mathrm{MET}_{2}$ relaxation when the size of the shortlived component is greater than approximately $4 \%$ of the total signal. 


\section{In vivo Magnetic Resonance}

Fig. 3 displays the results of the ROI-based $\mathrm{MET}_{2}$ analysis in vivo. Fig. 3a and $3 \mathrm{~b}$ show sample $T_{2}$ decay curves from normal and tumor ROIs and corresponding monoexponential fits (Eq. [2] with $J=1$ ). It can be seen that signal from tumor ROIs showed a larger deviation from a monoexponential model than signal from normal grey matter, suggesting a multiexponential model may be more appropriate for tumor signal. The $\mathrm{MET}_{2}$ results (Fig. $3 \mathrm{c}$ and $3 \mathrm{~d}$ ) from normal and tumor ROIs support this. For tumor ROIs, eight of eleven animals exhibited $T_{2}$ spectra with two components: I) a short-lived component $\left(T_{2, I}^{-}=20.7 \pm\right.$ $5.4 \mathrm{~ms})$ representing $6.8 \pm 6.2 \%$ of the signal and II) a long-lived component $\left(T_{2, I I}=76.4 \pm\right.$ $9.3 \mathrm{~ms}$ ) representing the remaining $93.2 \pm 6.2 \%$. In contrast, $99.4 \pm 1.3 \%$ of the total signal could be described by a single component $\left(T_{2}=48.8 \pm 2.3 \mathrm{~ms}\right)$ for normal grey matter, which is consistent with previous findings $(2,23)$. The relaxation times $\left(T_{2, n}\right)$ and signal fractions $\left(p_{n}\right)$ for each individual animal measured in vivo are detailed in Table 2.

Recall, images were smoothed with an anisotropic diffusion filter prior to voxel-based $\mathrm{MET}_{2}$ analysis. Fig. 4 displays the effects of this spatial smoothing on representative mean spectral $T_{2},\left\langle T_{2}\right\rangle$ (calculated using Eq. 3 over the entire spectral range, $t_{1} / 2<T_{2, j}<2 t_{N E}$ ), and $p_{I}$ maps. The $\left\langle T_{2}\right\rangle$ maps in Fig. $4 \mathrm{a}$ and $4 \mathrm{~b}$ illustrate the location of the tumor, which has a much longer $\left\langle T_{2}\right\rangle$ than surrounding tissue. The $p_{I}$ maps in Fig. $4 \mathrm{c}$ and $4 \mathrm{~d}$ illustrate that the anisotropic spatial smoothing employed results in decreased variability for $\mathrm{MET}_{2}$ results with minimal introduction of patial-voluming between tumor and normal tissue (though some information about tumor heterogeneity is likely lost after smoothing with this approach). Note, only results for voxels deemed admissible — defined here as voxels with spectra that exhibited two components in the range 8-200 ms - are shown. Additional contributions from white matter, which exhibited two components in this range consistent with previous findings $(1,2)$, were removed. These contributions were removed based upon the fact that the long-lived component in white matter has a $T_{2} \approx 50 \mathrm{~ms}$ (similar to the single component observed in grey matter shown in Fig. 3d), while the long-lived component in tumor tissue exhibited a $T_{2} \approx 75 \mathrm{~ms}$ (Component II in Fig. 3c). Thus, only spectra whose long-lived component exhibited a $T_{2}>60 \mathrm{~ms}$ are displayed in Fig. $4 \mathrm{c}$ and $4 \mathrm{~d}$ (and in the $p_{I}$ maps in Fig. 5).

Additional representative $\left\langle T_{2}\right\rangle$ and $p_{I}$ maps acquired in vivo are displayed in Fig. 5a (Rats \#9-11). Note that tumor voxels consistently exhibited biexponential decay, while normal grey matter voxels did not, which is consistent with the ROI-based results shown in Fig. 3. Mean ROI component values for the voxel-based $\mathrm{MET}_{2}$ results are summarized in Table 4 for all eleven animals. Only voxels deemed admissible within each ROI were used for these calculations. These results suggest that voxel-based $\mathrm{MET}_{2}$ analysis might be possible in vivo because: 1) the voxel-based results (Table 4) were similar to those obtained from ROI-based analysis (Table 2) for most animals and 2) $86.1 \pm 22.0 \%$ percent of voxels were deemed admissible. It should be noted that the ROI-based tumor signal was monoexponential in Rats \#6 and 7, while the voxel-based signal was multiexponential (for 30.0 and $69.2 \%$ of the voxels, respectively). This discrepancy can most likely be explained by partial-voluming of normal and tumor tissue within the chosen tumor ROIs. 


\section{Ex vivo Magnetic Resonance}

Fig. $3 \mathrm{e}$ and $3 \mathrm{f}$ displays the results of the ROI-based $\mathrm{MET}_{2}$ analysis ex vivo. For tumor ROIs, all three animals exhibited $T_{2}$ spectra with three components: I) a short-lived component $\left(T_{2, I}=37.8 \pm 6.5 \mathrm{~ms}\right)$ representing $7.8 \pm 6.5 \%$ of the signal, II) an intermediated-lived component $\left(T_{2, I I}^{-}=109.5 \pm 14.2 \mathrm{~ms}\right)$ representing $80.6 \pm 8.2 \%$ of the signal, and III $)$ a longlived component $\left(T_{2, I I I}=262.6 \pm 44.6 \mathrm{~ms}\right)$ representing the remaining $11.6 \pm 12.0 \%$ of the signal. Consistent with the in vivo findings, $94.5 \pm 5.3 \%$ of the total signal could be described by a single component $\left(T_{2}=51.2 \pm 8.4 \mathrm{~ms}\right)$ for normal grey matter ex vivo. It should be noted that relaxation times for components observed both in vivo and ex vivo (components I and II) were increased following fixation and washing in PBS, which is consistent with previous findings (24) in fixed primate grey matter. The relaxation times $\left(T_{2, n}^{-}\right)$and signal fractions $\left(p_{n}\right)$ for each sample measured ex vivo are detailed in Table 3.

In contrast to ROI-based measurements, voxel-based $T_{2}$ spectra in tumor consistently exhibited only two components ex vivo (though three components were resolved for some voxels). Fig. 5b displays $\left\langle T_{2}{ }^{-}\right\rangle$and $p_{I}$ maps acquired ex vivo and Table 4 summarizes these results. Again, only results for voxels whose spectra were deemed admissible — defined here as spectra that exhibited either two or three components in the range 15-300 ms - are shown in Fig. 5b. Additional contributions from white matter were also removed by only displaying spectra whose long-lived $T_{2}$ component was $>70 \mathrm{~ms}$ (using the same arguments as described above for in vivo measurements). These results suggest that voxel-based $\mathrm{MET}_{2}$ analysis might be possible with regards to resolving two components ex vivo because $97.6 \pm$ $4.1 \%$ percent of voxel within tumor ROIs were deemed admissible.

Comparing in vivo and ex vivo voxel-based results, it can be seen that similar results were found for Rats \#9 and \#10, while a substantial difference was found for Rat \#11. However, this particular animal (Rat \#11) was sacrificed a full four days after the in vivo study (compared to one day for the other animals). Therefore, comparison between the in vivo and ex vivo data in this case is difficult as tumor growth had progressed significantly in those four days. In summary, the presented ex vivo results argue against the assertion that the in vivo results are due to either partial-voluming or low SNR.

\section{DISCUSSION}

This study demonstrates that $T_{2}$ in a $\mathrm{C} 6$ rat glioblastoma model is heterogeneous on the scale of a typical MR voxel. Tumor signal exhibited two $T_{2}$ components in eight of the eleven animals in vivo, while a majority of the signal from contralateral grey matter could be described by a single $T_{2}$ component. Tumor signal in three of the eleven animals was monoexponential. Interestingly, the tumors of these animals were all relatively small in volume, suggesting that the observed $\mathrm{MET}_{2}$ signal might contain information about tumor progression. It should be noted, however, that no correlation was found between time postinnoculation and any of the $\mathrm{MET}_{2}$ parameters, which is not surprising given our observed variability in tumor volume across animals for a given time post-innoculation. Further longitudinal studies are needed to test the effect of tumor progression on $\mathrm{MET}_{2}$ parameters. 
A similar pattern was observed in the higher resolution and SNR ex vivo data; however, in this case three $T_{2}$ components were observed in tumor signal, while again a majority of the signal from contralateral gray matter could again be described by a single $T_{2}$ component. The reason an additional long-lived peak (component III in Fig. 3f) was resolved ex vivo is unclear. Previous studies in white matter (25) have shown that $T_{2}$ spectral characteristics are well-preserved in fixed samples. One possible explanation for the observed differences is the limited SNR of the in vivo data. In fact, spectra resulting from in vivo ROI-based analysis and ex vivo voxel-based analysis - data used for each of these analyses had approximately the same SNR - both exhibited two components (at least for a majority of tumor voxels in the ex vivo voxel-based analysis).

$\mathrm{MET}_{2}$ relaxation has been previously observed (6-10) in a number of tumor types and models with variable results. Given that microanatomical characteristics (and likely resultant relaxation spectra) vary across different tumor types and models, this observed variability is not surprising. Furthermore, a variety of models (e.g., biexponential, continuous) were employed in these past studies, which may be an additional contributing factor in this observed variability. In the current study, a linear least-squares algorithm with non-negative constraints (NNLS) was employed, which fits the data to a continuous distribution of decaying exponentials. This approach is particularly attractive as it makes no assumptions about the number of exponential components in the sample. Data sampling parameters (e.g, SNR, echo spacing) are an important consideration when employing such an approach (16). In the current study, Monte Carlo simulations were performed to ensure that these were sufficient to obtain reproducible results.

A number of physiological and microanatomical parameters are altered in tumors that may affect $T_{2}$. This list includes variations in $\mathrm{pO}_{2}$ (26), $\mathrm{pH}$ (27), and water content (28) as well as the presence (or absence) of irregular vasculature, necrotic tissue (29), or densely packed proliferating cells (30). Given the heterogeneity common in tumors, a typical tumor voxel may contain regions that vary substantially in several of these parameters, which may explain the observed $\mathrm{MET}_{2}$ relaxation. The relevance of each of these with respect to the findings presented herein is discussed below.

It has been known for some time that the mean $T_{2}$ (as well as $T_{1}$ ) of viable tumor is elevated relative to normal tissue (31) and that this increase correlates with increased water content (28). This is consistent with the findings presented herein, in which $\left\langle T_{2}\right\rangle$ was found to be elevated in tumors relative to contralateral grey matter. This can be attributed to the fact that the long-lived $T_{2}(76.4 \pm 9.3 \mathrm{~ms})$ component in tumor signal, which represented a majority of the total signal $(93.2 \pm 6.2 \%)$, was longer than that observed in contralateral grey matter $(48.8 \pm 2.3 \mathrm{~ms})$. Therefore, it is reasonable to assume that the long- $T_{2}$ component in tumor signal represents signal from viable tumor tissue and that the underlying mechanism for the increased $T_{2}$ of the component is increased water content. It should be noted that additional factors may also play a role in the elevated $T_{2}$ of this component. For example, hypoxia (low tissue $\mathrm{pO}_{2}$ ) and intracellular acidosis have been shown to result in increased $T_{2}$ in skeletal muscle (32). Given that viable tumor cells are commonly hypoxic and acidic, these may be underlying factors contributing to the observed long- $T_{2}$ component in this study. 
Assignment of an underlying mechanism for the short- $T_{2}$ component observed in tumor signal is less straightforward. Recall the $T_{2}$ of this component was substantially reduced $(20.7 \pm 5.4 \mathrm{~ms})$ relative to contralateral grey matter $(48.8 \pm 2.3 \mathrm{~ms})$; therefore, it is unlikely that this component reflects partial-volumed normal tissue within tumor ROIs (or voxels). One possible explanation for the observed short- $T_{2}$ component in tumor signal is the presence of densely packed proliferating cells. It has previously been shown (33) that $T_{2}$ and cellularity are inversely related in a human melanoma xenograft model — most likely attributed to that fact that macromolecular content increases and water content decreases with increased cellularity. The authors noted that $T_{2}$ varied by a factor of approximately 1.5 between regions with extracellular volume fractions of 5 and 70\% (27). In terms of the current study, it appears that increased cellularity alone cannot explain the observed short- $T_{2}$ component. Even if we assume that the extracellular volume fraction varies to the extreme of the previously mentioned study, this variation alone could not explain the approximately 2.5-fold decrease in $T_{2}$ of the short- $T_{2}$ component in tumor signal relative to contralateral grey matter. Another possible explanation for the observed short- $T_{2}$ component in tumor signal is the presence of irregular tumor vasculature (e.g., dead ends, shunts that are commonly found in tumor associated vasculature (29)), which may result in accumulation of paramagnetic deoxyhemoglobin and a corresponding decrease in $T_{2}$ for adjacent tissue. A final, more likely, explanation for this component is the presence of necrotic tissue, which has been shown to have decreased water content (and similar $T_{2}$ ) relative to normal tissue in surgical specimens taken from patients with lung cancer (8). A longitudinal study (7) in an EO 771 adenocarcinoma mouse model also noted decreases in $T_{2}$ that correlated with the onset of necrosis.

Additional studies are needed to determine the physiological origin of these components. One proposed study is to use dynamic contrast-enhanced studies (DCE) MRI and histopathology and assess tumor necrosis. Correlation of these measures to the short-lived $T_{2}$ signal fraction will allow us to test the hypothesis that this component arises from necrotic tissue. Though the underlying physiological origin of the observed $\mathrm{MET}_{2}$ components is currently unknown, $\mathrm{MET}_{2}$ analysis holds promise as a non-invasive tool for characterizing the tumor microenvironment in vivo on a sub-voxel scale.

\title{
Acknowledgments
}

Grant Sponsors: NIH EB001744, U24 CA126588 (NCI Small Animal Imaging Resource Program), 1K25 EB005936, 1K99CA127599-01 and NSF Career Award 0448915 (M.D.D). NIH P30 CA68485 (Cancer Center Support Grant)

We thank Jarrod True for providing expert assistance and guidance in technical animal care and handling issues and Kevin Wilson for assistance in MATLAB code development.

\section{Abbreviations used}

\author{
MET $_{2}$ multiexponential $T_{2}$ \\ SNR signal-to-noise ratio \\ ROI region of interest
}


NNLS non-negative least-squares

$\Delta$ TE $\quad$ interecho spacing

NE number of echoes

NEX number of averaged excitations

DCE dynamic contrast-enhanced

\section{References}

1. Stewart WA, Mackay AL, Whittall KP, Moore GRW, Paty DW. Spin-spin relaxation in experimental allergic encephalomyelitis: Analysis of CPMG data using a nonlinear least-squares method and linear inverse-theory. Magn Reson Med. 1993; 29:767-775. [PubMed: 8350719]

2. Mackay A, Whittal K, Adler J, Li D, Paty D, Graeb D. In-vivo visualization of myelin water in brain by magnetic resonance. Magn Reson Med. 1994; 31:673-677. [PubMed: 8057820]

3. Does MD, Snyder RE. T2 relaxation of peripheral nerve measured in-vivo. Magn Reson Imaging. 1995; 13:575-580. [PubMed: 7674853]

4. Vasilescu V, Katona E, Simplaceanu V, Demco D. Water compartments in myelinated nerve. 3. Pulsed NMR results. Experientia. 1978; 34:1443-1444. [PubMed: 309823]

5. Saab G, Thompson RT, Marsh GD. Multicomponent T2 relaxation of in vivo skeletal muscle. Magn Reson Med. 1999; 42:150-157. [PubMed: 10398961]

6. Kroeker RM, Stewart CA, Bronskill MJ, Henkelman RM. Continuous distributions of NMR relaxation times applied to tumors before and after therapy with X-rays and cyclophosphamide. Magn Reson Med. 1988; 6:24-36. [PubMed: 3352503]

7. Kovalikova Z, Hoehn-Berlage MH, Gersonde K, Porschen R, Mittermayer C, Franke RP. Agedependent variation of $\mathrm{T} 1$ and $\mathrm{T} 2$ relaxation times of adenocarcinoma in mice. Radiology. 1987; 164:543-548. [PubMed: 3602399]

8. Shioya S, Haida M, Ono Y, Fukuzaki M, Yamabayashi H. Lung cancer: differentiation of tumor, necrosis, and atelectasis by means of T1 and T2 values measured in vitro. Radiology. 1988; 167:105-109. [PubMed: 3347709]

9. Fantazzini P, Sarra A. A comparison of the proton relaxation in human epithelial tumors and associated uninvolved tissue. MAGMA. 1994; 2:405-407.

10. Schad LR, Brix G, Zuna I, Harle W, Lorenz WJ, Semmler W. Multiexponential proton spin-spin relaxation in MR imaging of human brain tumors. J Comput Assist Tomogr. 1989; 13:577-587. [PubMed: 2545751]

11. Carr HY, Purcell EM. Effects of Diffusion on Free Precession in Nuclear Magnetic Resonance Experiments. Physical Review. 1954; 94:630.

12. Meiboom S, Gill D. Modified Spin-Echo Method for Measuring Nuclear Relaxation Times. Rev Sci Instrum. 1958; 29:688-691.

13. Bonny JM, Boespflug-Tanguly O, Zanca M, Renou JP. Multi-exponential analysis of magnitude MR images using a quantitative multispectral edge-preserving filter. J Magn Reson. 2003; 161:2534. [PubMed: 12660108]

14. Lawson, CL.; Hanson, RJ. Solving least squares problems. Englewood Cliffs, NJ: Prentice-Hall; 1974.

15. Whittall KP, Mackay AL. Quantitative Interpretation of NMR Relaxation Data. J Magn Reson. 1989; 84:134-152.

16. Graham SJ, Stanchev PL, Bronskill MJ. Criteria for analysis of multicomponent tissue T2 relaxation data. Magn Reson Med. 1996; 35:370-378. [PubMed: 8699949]

17. Barth RF. Rat brain tumor models in experimental neuro-oncology: the 9L, C6, T9, F98, RG2 (D74), RT-2 and CNS-1 gliomas. J Neurooncol. 1998; 36:91-102. [PubMed: 9525831] 
18. Poon CS, Henkelman RM. Practical T2 quantitation for clinical applications. J Magn Reson Imaging. 1992; 2:541-553. [PubMed: 1392247]

19. Levitt MH, Freeman R. Compensation for pulse imperfections in NMR spin-echo experiments. J Magn Reson. 1981; 43:65-80.

20. Karlsen OT, Verhagen R, Bovee WMMJ. Parameter estimation from Rician-distributed data sets using a maximum likelihood estimator: Application to T1 and perfusion measurements. Magn Reson Med. 1999; 41:614-623. [PubMed: 10204887]

21. Gerig G, Kubler O, Kikinis R, Jolesz FA. Nonlinear anisotropic filtering of MRI data. IEEE Trans Med Imaging. 1992; 11:221-232. [PubMed: 18218376]

22. Jones CK, Whittall KP, MacKay AL. Robust myelin water quantification: averaging vs. spatial filtering. Magn Reson Med. 2003; 50:206-209. [PubMed: 12815697]

23. Does MD, Gore JC. Compartmental study of T1 and T2 in rat brain and trigeminal nerve in vivo. Magn Reson Med. 2002; 47:274-283. [PubMed: 11810670]

24. D'Arceuil HE, Westmoreland S, de Crespigny AJ. An approach to high resolution diffusion tensor imaging in fixed primate brain. Neuroimage. 2007; 35:553-565. [PubMed: 17292630]

25. Laule C, Kozlowski P, Leung E, Li DK, Mackay AL, Moore GR. Myelin water imaging of multiple sclerosis at 7 T: correlations with histopathology. Neuroimage. 2008; 40:1575-1580. [PubMed: 18321730]

26. Padhani AR, Krohn KA, Lewis JS, Alber M. Imaging oxygenation of human tumours. Eur Radiol. 2007; 17:861-872. [PubMed: 17043737]

27. Gatenby RA, Gillies RJ. Why do cancers have high aerobic glycolysis? Nat Rev Cancer. 2004; 4:891-899. [PubMed: 15516961]

28. Kiricuta IC Jr, Simplaceanu V. Tissue water content and nuclear magnetic resonance in normal and tumor tissues. Cancer Res. 1975; 35:1164-1167. [PubMed: 1120306]

29. Bergers G, Benjamin LE. Angiogenesis: Tumorigenesis and the angiogenic switch. Nat Rev Cancer. 2003; 3:401-410. [PubMed: 12778130]

30. Krohn KA, Mankoff DA, Eary JF. Imaging cellular proliferation as a measure of response to therapy. J Clin Pharmacol. 2001:96S-103S. [PubMed: 11452736]

31. Damadian R. Tumor detection by nuclear magnetic resonance. Science. 1971; 171:1151-1153. [PubMed: 5544870]

32. Damon BM, Gregory CD, Hall KL, Stark HJ, Gulani V, Dawson MJ. Intracellular acidification and volume increases explain R(2) decreases in exercising muscle. Magn Reson Med. 2002; 47:14-23. [PubMed: 11754438]

33. Jakobsen I, Lyng H, Kaalhus O, Rofstad EK. MRI of human tumor xenografts in vivo: proton relaxation times and extracellular tumor volume. Magn Reson Imaging. 1995; 13:693-700. [PubMed: 8569443] 


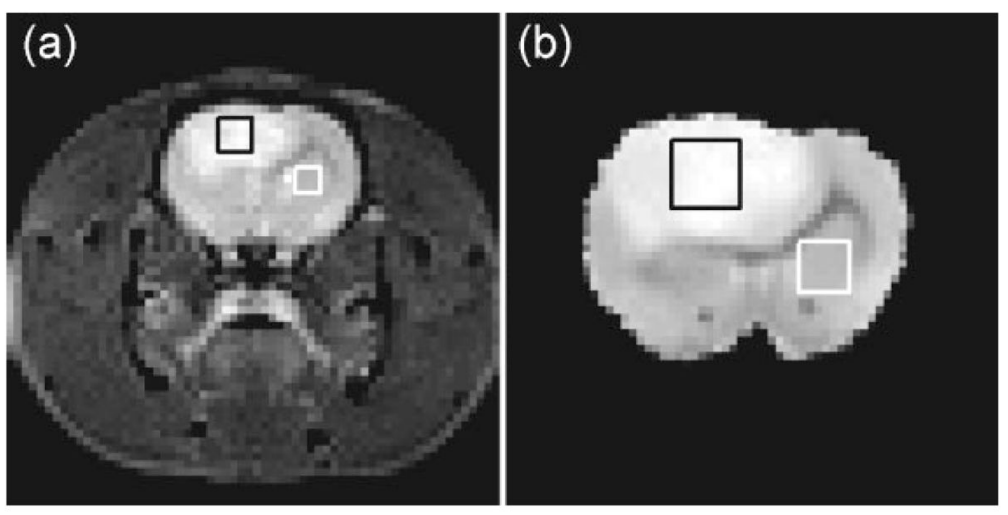

Figure 1.

(a) Representative $T_{2}$-weighted images acquired in vivo $(\mathrm{TE}=40 \mathrm{~ms}$ ) along with representative ROIs for tumor (black box) and normal (contralateral subcortical grey matter, white box) tissue. (b) Corresponding ex vivo image ( $\mathrm{TE}=30 \mathrm{~ms}$ ) from the same animal (Rat \#10). 

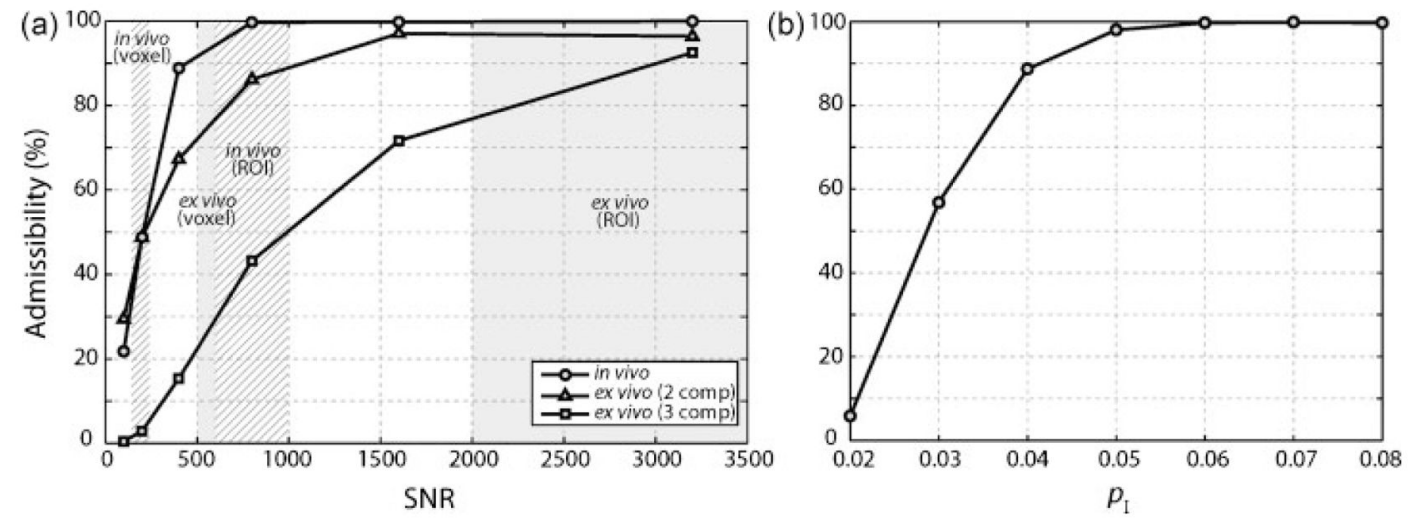

Figure 2.

(a) Admissibility as a function of SNR for simulated in vivo and ex vivo tumor $\mathrm{MET}_{2}$ data (using Eq. [1] and the parameters listed in Table 1). For ex vivo data, the admissibility for resolving all three observed components ( 3 comp) as well as resolving the short-lived component from the two longer-lived components ( 2 comp) was tabulated. The approximate range of experimental SNRs for voxel- and ROI-based analyses in vivo (hatched boxes) and ex vivo (grey boxes) is also indicated. (b) Admissibility as a function of $p_{I}$ for simulated in vivo data $(\mathrm{SNR}=800)$. 

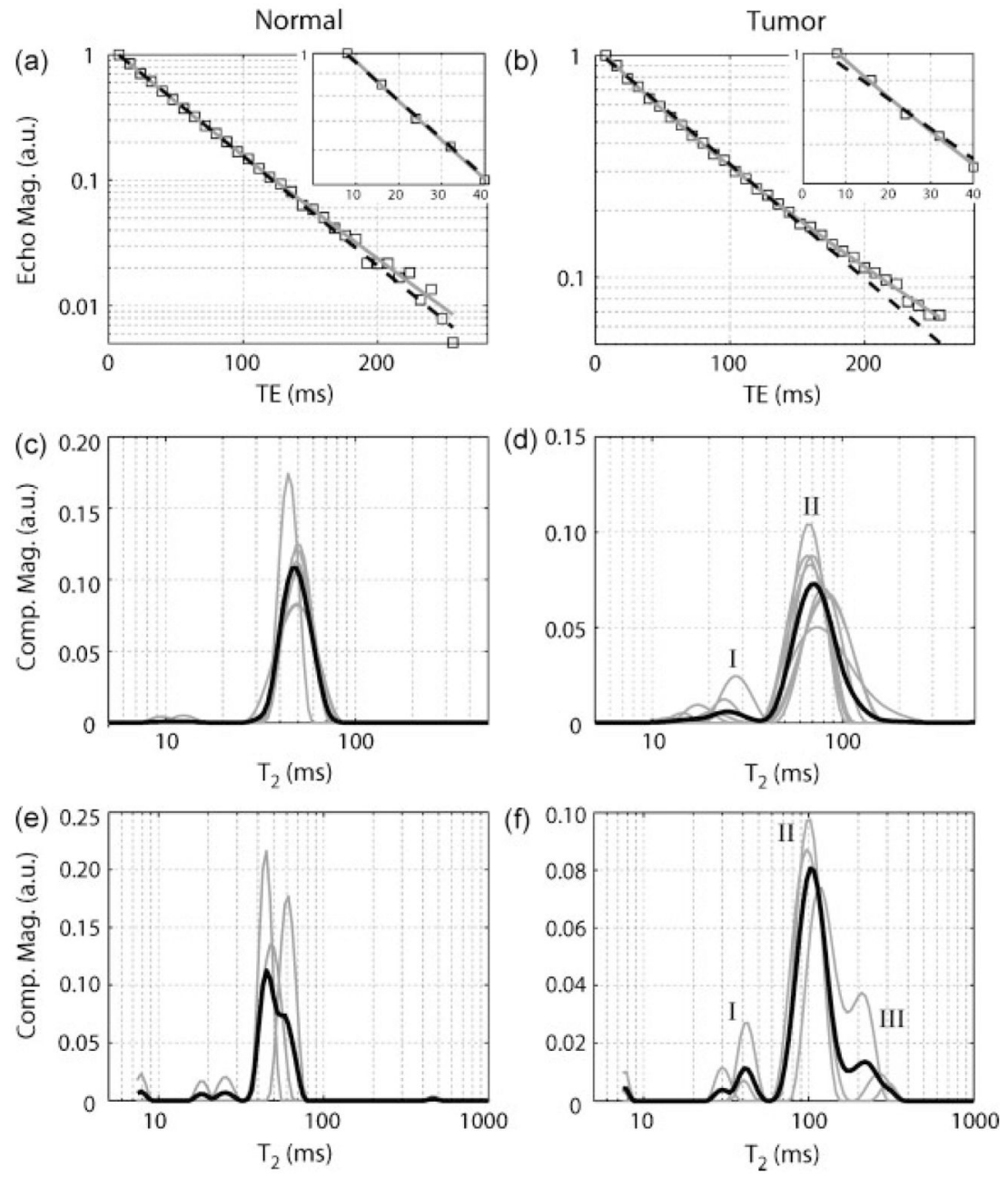

Figure 3.

( $a$ and b) Sample decay curves from normal and tumor tissue ROIs in vivo. Data were fitted using multiexponential (grey solid line; Eq. [2] with J=100) and monoexponential (black dashed line; Eq. [2] with $\mathrm{J}=1$ and $\mu=0$ ) models. Note that tumor signal deviates from the monoexponential model fit, especially at shorter echo times as shown in the zoomed insets. (c and d) $T_{2}$ spectra from in vivo measurements for individual animals (gray) as well as the mean spectrum across animals (black). Two components (I and II) were observed in tumor signal in eight of the eleven rats in vivo (only spectra from these eight rats are given here), while signal from normal tissue was consistently monoexponential. (e and f) $T_{2}$ spectra from ex vivo measurements. Three components (I, II, and III) were in observed in tumor signal in all three rats. Once again, normal tissue was consistently monoexponential. Note, the small peaks to the far left of these spectra were deemed artifacts of the fitting process and were ignored in subsequent analysis. Component values for each rat are listed in Tables 2 and 3 for in vivo and ex vivo measurements, respectively. 


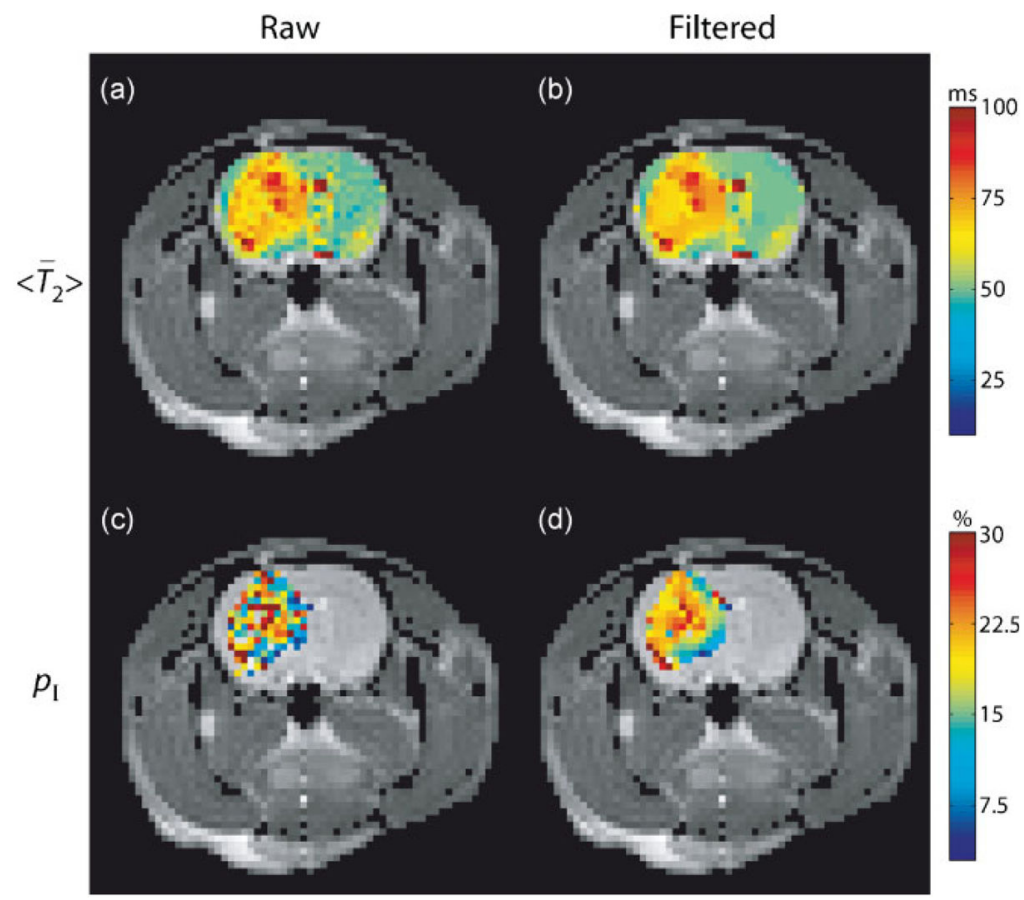

Figure 4.

$\left\langle T_{2}\right\rangle$ (a and b) and $p_{I}$ (c and d) maps calculated from raw and filtered images overlaid onto an anatomical image from one animal (Rat \#3). For $p_{I}$, only voxels whose $T_{2}$ spectra were deemed admissible - defined as spectra that exhibited two components in the range 8-200 $\mathrm{ms}$ - are shown. Additional contributions from white matter were also removed (see Methods). Observe that there exists significant intra-tumoral heterogeneity. 


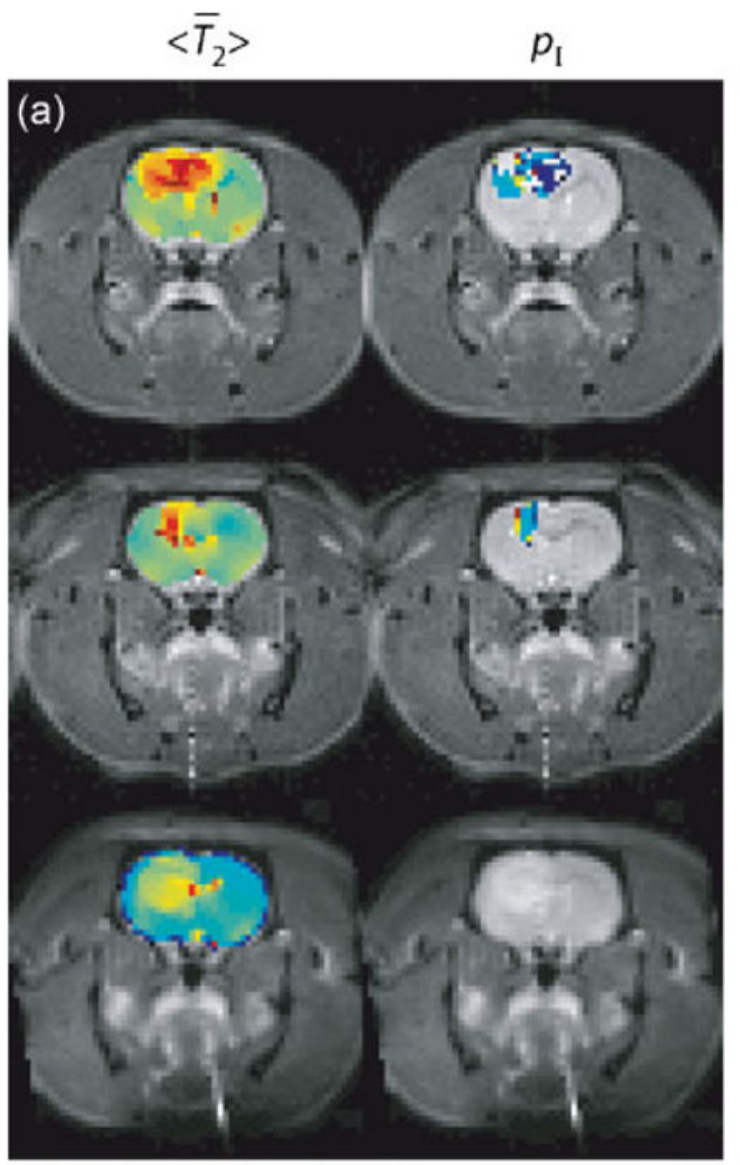

$\mathrm{ms}$

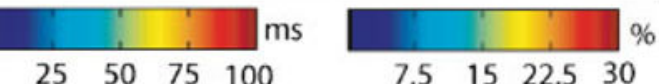

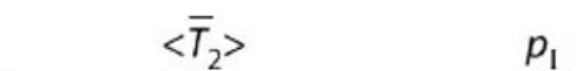

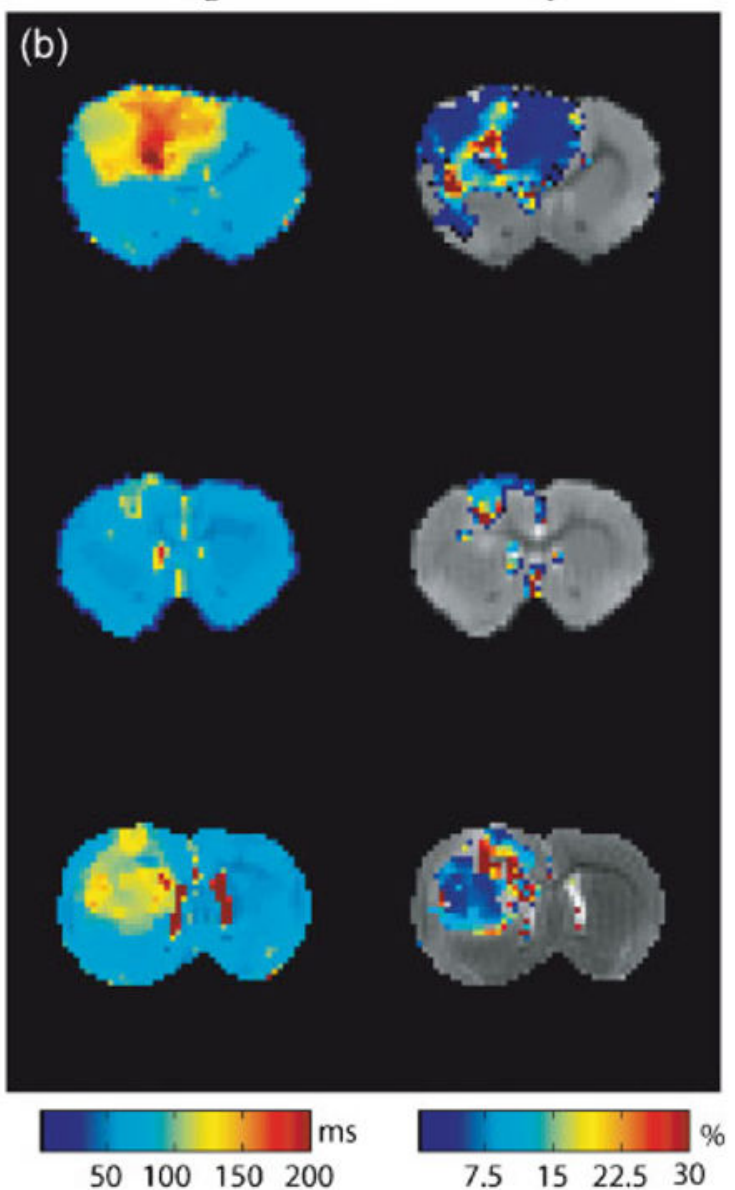

Figure 5.

(a) In vivo $\left\langle T_{2}\right\rangle$ and $p_{I}$ maps overlaid onto anatomical images (Rats \#9-11). (b)

Corresponding ex vivo results from the same three rats. For $p_{I}$, only voxels whose $T_{2}$ spectra were deemed admissible — defined as spectra that exhibited two components in the range 8-200 ms or 15-300 ms for in vivo and ex vivo acquisition, respectively - are shown. Additional contributions from white matter were also removed (see Methods). 


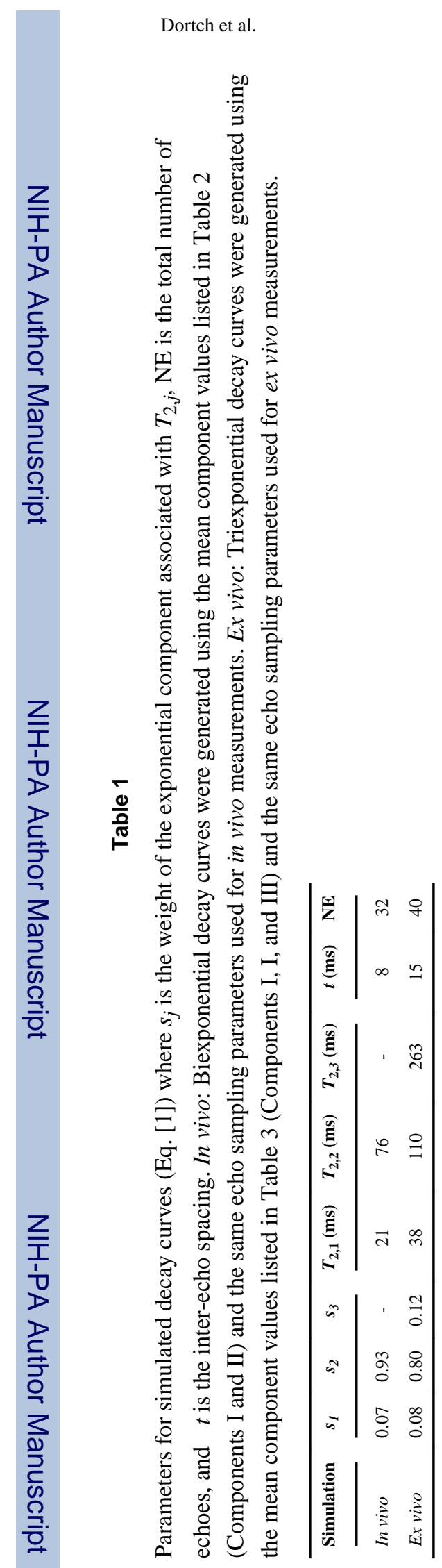

NMR Biomed. Author manuscript; available in PMC 2014 September 30. 


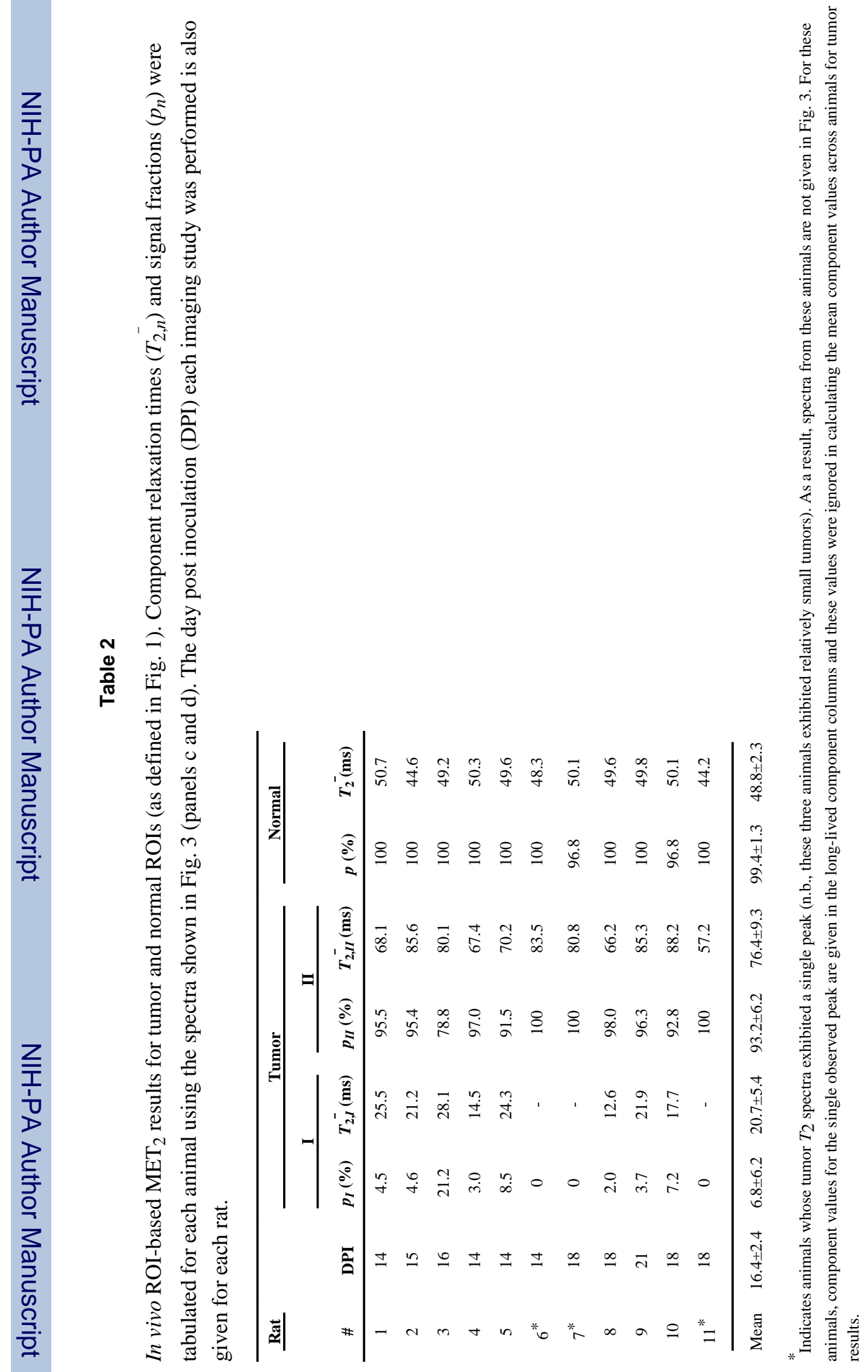




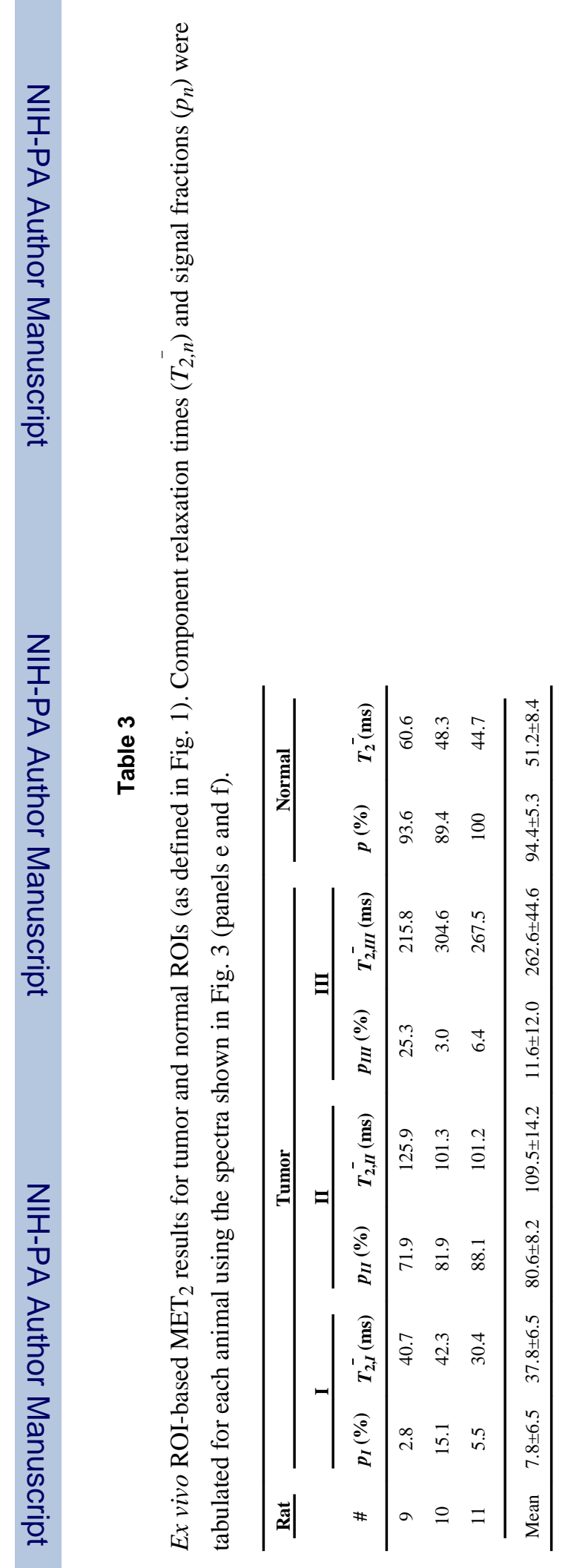




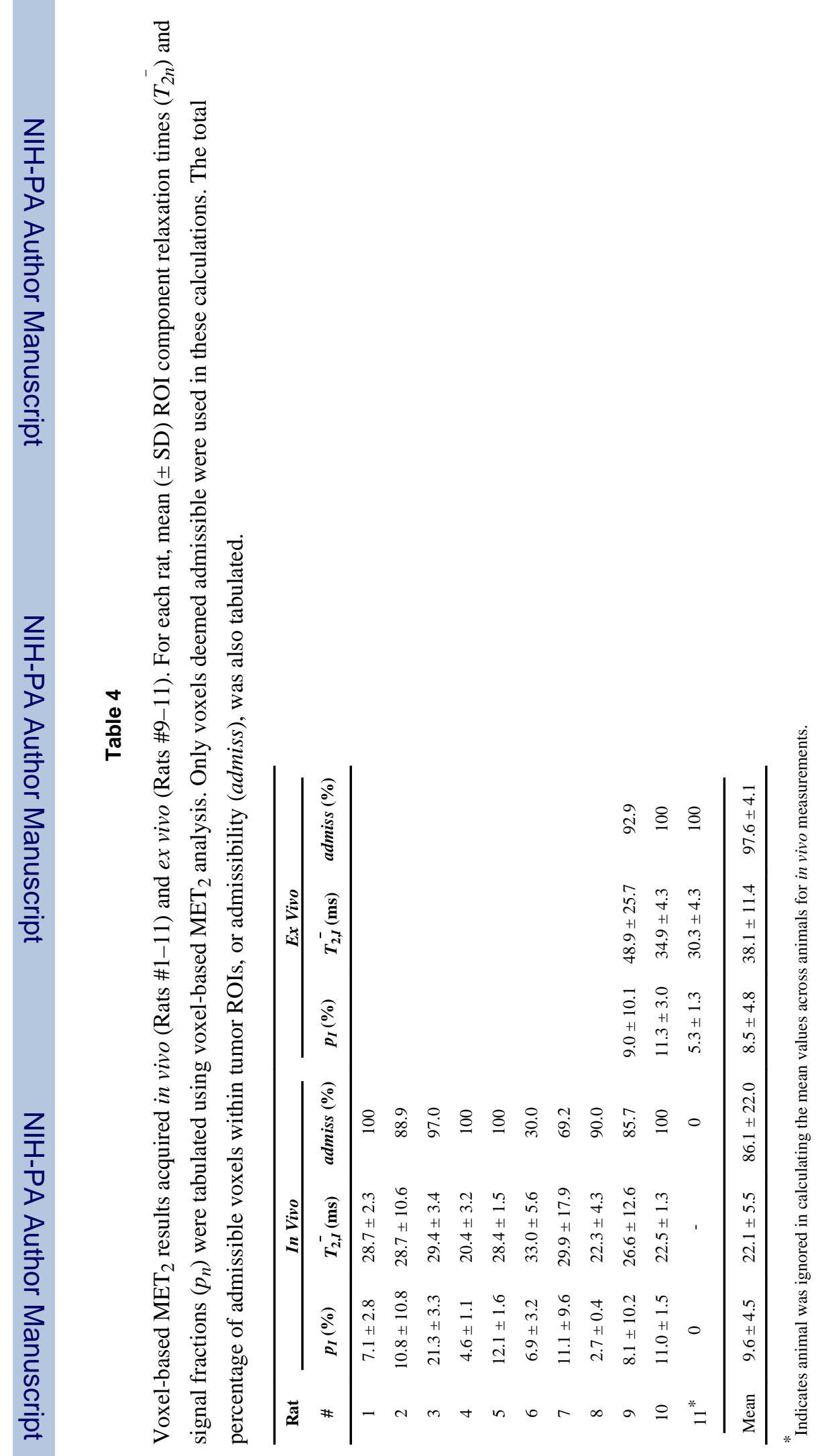

